\title{
Calculation of the thickness and optical constants of lead titanate thin films grown on MgO from their transmission spectra
}

\author{
A. Essahlaoui ${ }^{1, *}$ H. Essaoudi ${ }^{2}$, A. Hallaoui ${ }^{1}$, M. Bouhadda ${ }^{3}$, A. Labzour ${ }^{1}$, A. Housni ${ }^{4}$ \\ ${ }^{1}$ Engineering Sciences Laboratory (LSI), Multidisciplinary Faculty of Taza, Sidi Mohamed Ben Abdellah University, B.P. \\ 1223, Taza gare, Taza, Morocco. \\ ${ }^{2}$ Faculty of Sciences, Moulay Ismail University, B.P.11201, Zitoune, Meknes, Morocco. \\ ${ }^{3}$ MACS Laboratory, Moulay Ismail University, Meknes, Morocco. \\ ${ }^{4}$ Higher Institute of Nursing Professions and Health Techniques of Fes-Fes-Morocco
}

Received 28 Feb 2017,

Revised 28 Sep 2017,

Accepted 30 Sep 2017

Keywords

$\checkmark$ Thin films;

$\checkmark$ Optical constants;

$\checkmark$ Band gap;

$\checkmark$ Transmission;

$\checkmark$ Refractive index

A. Essahlaoui

abdelouahed.essahlaoui@usmba.ac.ma $\mathbf{+ 2 1 2 6 7 7 7 6 7 0 7 0}$

\begin{abstract}
In this paper, the optical constants and thickness of thin lead titanate $\left(\mathrm{PbTiO}_{3}\right)$ films are investigated. The studied films are grown by pulsed laser deposition on $\mathrm{MgO}$ substrate. The interference modulated transmission spectra $\mathrm{T}(\lambda)$ at normal incidence were obtained in the spectral region from $300 \mathrm{~nm}$ up to 1000 $\mathrm{nm}$. The presented films have been characterized using the extremes of the interference fringes in accordance with the straightforward method proposed by Swanepoel which is applied to derive the real and imaginary parts of the complex index of refraction and also the film thickness. We have therefore determined the dispersion of the real part of the refractive index, the absorption and extinction coefficients. Furthermore, the energy band gap has been determined from the absorption coefficient values using the direct transition model proposed by Tauc.
\end{abstract}

\section{Introduction}

Piezoelectric and ferroelectric materials are very present around us and are an integral part of our lives. In cars, fuel consumption is optimized by piezoelectric injectors and safety is enhanced with airbags controlled by accelerometers. We use, store and transport the digital data with non-volatile ferroelectric memories in hard drives (SSD) and USB keys (EEPROM flash memory). We communicate using smartphones equipped with touch screens and using hyper-frequency filters (allowing the data transfer to the relay antennas) also using ferro- and piezoelectric materials.

As a result, technological and economic constraints have arisen which has pusched manufacturers to miniaturize the devices more and more and the research has developed on integrated ferro and piezoelectric materials of increasingly reduced size, mainly in thin films form. Materials based on $\mathrm{Pb}$ such as $\mathrm{PbTiO} 3$ are the best piezoelectric and ferroelectric materials families suitable for devices integration such as non-volatile memories [1], Infra-red sensors [2,3], dielectric properties [4] and terahertz transmission filter [5]. $\mathrm{PbTiO}_{3}(\mathrm{PT})$ is one of the more interesting and more studied peroveskites possessing a ferroelectric phase under ambient conditions [6]. PT has large electro-optic coefficient and high photorefractive sensitivity, so it can be used as an optical sensor and, more recently, optical waveguide devices.

Recently, the photovoltaic properties of $\mathrm{PbTiO} 3$ Thin films are studied by determination of photovoltage, photocurrent density and fill factor [7]. The magnetoelectric coupling at the epitaxial ferromagnetic/ferroelectric $(\mathrm{Ni} / \mathrm{PbTiO} 3)$ is also studied; and it demonstrated that it depends strongly on the bonding mechanism and the type of magnetic interaction among the interfacial atoms [8].

Lead titanate thin films, deposited on adequate substrate, are an important material for nonlinear devices. These devices need a film with specific thickness and it is important to know the refractive index and absorption coefficient as function of a wavelength to predict the photoelectric behavior of a device. Knowledge of these optical constants is also necessary to determine the optical band gap. 
The thickness of thin films and refractive index can be determined using m-lines method $[9,10]$ and ellipsometry spectroscopic $[11,12]$. Unfortunately, these techniques require significant experimental equipment. In this work, we will report the results of $\mathrm{PbTiO}_{3}$ thin films characterizations using the interference fringes (intensity modulation) which are obtained in transmission spectra. Generally, the spectrum of transmittance can roughly be divided into four regions:

- Transparent region when the transmission is characterized through multiple reflections.

- Region of weak absorption when absorption coefficient $\alpha$ is small but starts to reduce the transmission.

- Region of medium absorption when $\alpha$ is large and the transmission decreases.

- Region of strong absorption when the transmission decreases drastically due almost exclusively to the effect of $\alpha$.

In this paper, the determination of optical properties of grown thin films was performed using only their transmission spectra [13-15]. Specifically, the dispersion of refractive index, extinction coefficient and absorption coefficient as a function of the wavelength $\mathrm{n}(\lambda), \mathrm{k}(\lambda)$ and $\alpha(\lambda)$, respectively, are estimated. Moreover, the energy band gap value is also calculated.

\section{Sample Preparation :}

The waveguiding thin films of lead titanate have been deposited on substrate (100) oriented $\mathrm{MgO}$ by laser ablation technique [16]. The choice of $\mathrm{MgO}$ as a substrate is based on three reasons: its refractive index is lower than that of the PT, hence the feasibility of a planar waveguides, the small difference of the lattice mismatching (7.7\% [17]) and the thermal expansion coefficients [18] allows epitaxial growth with good PT/ MgO interface. A laser ablation system was equipped with a quadrupled frequency Nd-YAG laser (266 nm laser wavelength) with a repetition rate of $5 \mathrm{~Hz}$ and a pulse duration of $12 \mathrm{~ns}$. The focusing diameter of the laser beam is approximately $1 \mathrm{~mm}$ and the energy is adjusted to obtain a fluence approximately $1.5 \mathrm{~J} / \mathrm{cm}^{2}$. The substrate is located at $3.5 \mathrm{~cm}$ from the target and the growing temperature is $700{ }^{\circ} \mathrm{C}$. The deposition is carried out under 0.4 mbar oxygen. The PbTiO3 target is a commercial hot pressed ceramic (Inland Europe, Praxair surface technologies, purity $99.99 \%$ ). Oxygen pressure influences the static oxidation processes of the growing thin films and control the relative stability of the oxygened species in the plasma while the temperature is an important parameter for obtaining the stoichiometric PT films with a purely perovskite structure[19-21].

\section{Calculation Method, Results and Discussion :}

\subsection{Calculation of the refractive index and film thickness}

The optical system under consideration corresponds to homogeneous and uniform thin films, deposited on thick transparent substrates. The film has thickness $d$ and complex refractive index $n_{c}=n-i k$, where $n$ is the refractive index and $\mathrm{k}$ the extinction coefficient. The substrate thickness is several orders of magnitude larger than $\mathrm{d}$, and its refractive index is symbolised by $s$ which the dispersion is given by the following relationship [22]:

$$
s^{2}=a+\frac{b \cdot \lambda^{2}}{\lambda^{2}-c}
$$

where $a=1.3224, b=1.6294, c=0.0138$ and $\lambda$ is the wavelength $(\mu \mathrm{m})$.

The system is surrounded by air with refractive index $n_{a}=1$. Taking all the multiple reflections at the three interfaces into account, it can be shown that in the case $\mathrm{k}^{2}<<\mathrm{n}^{2}$, the transmission $\mathrm{T}$ at normal incidence is given by [23] :

$$
T=\frac{A x}{B-C x \cos \varphi+D x^{2}}
$$

Where

$$
\begin{aligned}
& A=16 n^{2} s \\
& B=(n+1)^{3}\left(n+s^{2}\right) \\
& C=2\left(n^{2}-1\right)\left(n^{2}-s^{2}\right) \\
& D=(n-1)^{3}\left(n-s^{2}\right) \\
& \varphi=\frac{4 \pi n d}{\lambda} \\
& x=\exp (-\alpha d) \\
& k=\frac{\alpha \lambda}{4 \pi}
\end{aligned}
$$


The transmission values at the maxima and minima of the interference fringes can be obtained from equation (2) by setting the interference condition $\cos \varphi=+1$ for maxima $\left(T_{\max }\right)$ and $\cos \varphi=-1$ for minima $\left(\mathrm{T}_{\min }\right)$. The expressions of $\mathrm{T}_{\max }$ and $\mathrm{T}_{\min }$ are the following:

$$
T_{\max }=\frac{A x}{B-C x+D x^{2}}(10) \text { and } T_{\min }=\frac{A x}{B+C x+D x^{2}}
$$

From these two new formulas, many equations that provide the basis of the method in use are easily derived. Figure 1 shows the measured transmittance $(\mathrm{T})$, the obtained envelopes, $\mathrm{T}_{\max }$ and $\mathrm{T}_{\min }$ in the spectral region with interference fringes for the $\mathrm{PbTiO}_{3}$ thin film grown on $\mathrm{MgO}$ single crystal substrate. The creating of the envelopes of interference maxima and minima is in accordance to Swanepoel's method [22] which is based on the idea of Manifacier and al. [24]. The spectrum $T(\lambda)$ is experimentally obtained under normal incidence.

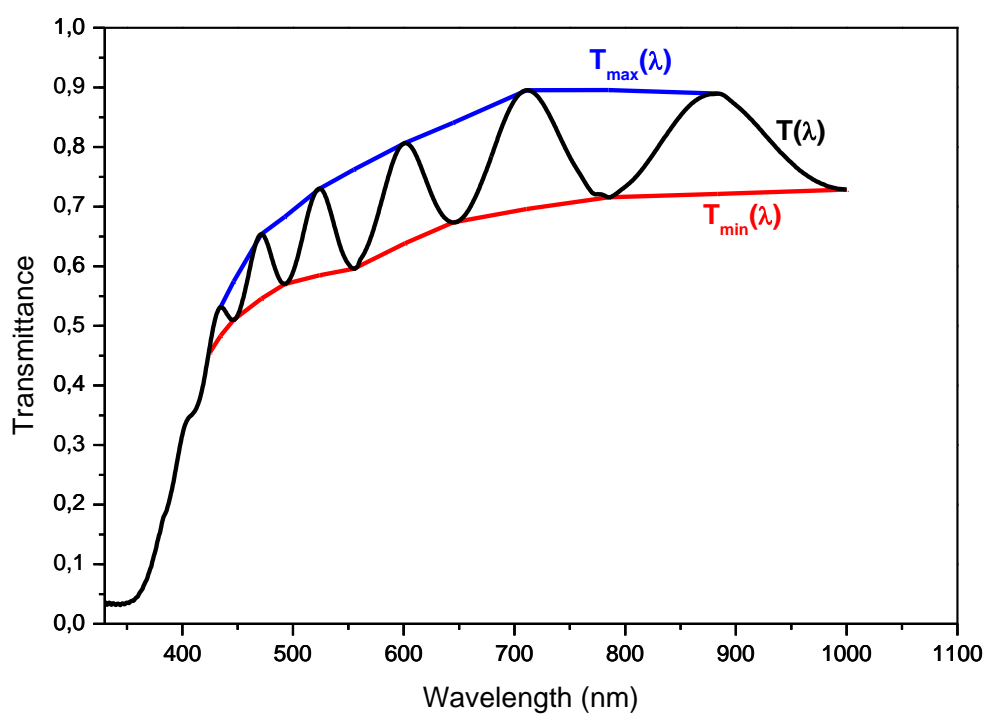

Figure 1: Optical transmission spectra of $\mathrm{PbTiO}_{3}$ deposited on $\mathrm{MgO}$ substrate. Two envelopes $\mathrm{T}_{\max }$ and $\mathrm{T}_{\min }$ are formed by connecting the maxima and minima respectively.

We mainly observe two distinct regions:

$\circ$ A region (500 to $950 \mathrm{~nm}$ ) having interference fringes whose operation allows us to determine both films refractive index and thickness.

- A region (350 to $500 \mathrm{~nm}$ ) where the absorption becomes larger, which results in a progressive damping of the interference fringes and a strong decrease of the transmittance.

The first approximate value of the real part of the refractive index $n$ of the film, in the spectral region of medium and weak absorption can be calculated using following expression:

$$
\begin{gathered}
\qquad n=\left[M+\left(M^{2}-s^{2}\right)^{\frac{1}{2}}\right]^{\frac{1}{2}} \\
\text { Where } M=\frac{2 s\left(T_{\max }-T_{\min }\right)}{T_{\text {max }} T_{\text {min }}}-\frac{s^{2}+1}{2}
\end{gathered}
$$

In the last equation, $\mathrm{T}_{\max }$ and $\mathrm{T}_{\min }$ are the transmission maximum and the corresponding minimum at a certain wavelength $\lambda$. Alternatively, one of these values is an experimental interference maximum (or minimum) and the other one is derived from the corresponding envelope at the same wavelength $\lambda$. The values of refractive index $\mathrm{n}_{1}$, which is the first approximate value of the real part of the refractive index, as calculated from equation (12) are shown in table 2.

The accuracy of this initial estimation of the refractive index is improved after calculating $\mathrm{d}$, as will be explained below. Now, it is necessary to take into account the basic equation for interference fringes:

$$
2 \mathrm{nd}=\mathrm{m} \lambda
$$

Where the order number $\mathrm{m}$ is an integer for maxima and half integer for minima. Moreover, if $\mathrm{n}\left(\lambda_{1}\right)$ and $\mathrm{n}\left(\lambda_{2}\right)$ are the refractive indices at two adjacent maxima (or minima) at $\lambda_{1}$ and $\lambda_{2}$, it follows that the film thickness is given by: 


$$
d=\frac{\lambda_{1} \lambda_{2}}{2 \cdot\left(\lambda_{1} n\left(\lambda_{2}\right)-\lambda_{2} n\left(\lambda_{1}\right)\right)}
$$

Equation (15) is very sensitive to errors in refractive index $n$ and is not very accurate. The film thickness values obtained from equation (15) are shown as $d_{1}$ in table 2 . There is some dispersion in the values of $d_{1}$. This deviation is an indication that $\mathrm{n}_{1}$ as calculated from equation (12) is not accurate enough.

The average value of thickness is $d_{1}=525 \pm 80 \mathrm{~nm}$. This value of $d_{1}$ can now be used, along with $n_{1}$, to calculate the 'order numbers' $\mathrm{m}_{0}$ for different extremes using equation (14). The accuracy of thickness values can now be significantly increased by taking the corresponding exact integer or half integer values of $\mathrm{m}$ associated with each extreme point and deriving a new thickness $d_{2}$ from equation (14) again using values of $n_{1}$. The thickness values found in this way have a smaller dispersion and the average value of $\mathrm{d}_{2} 522 \pm 9 \mathrm{~nm}$. Then, using the accurate values of $\mathrm{m}$ and $\mathrm{d}_{2}$, refractive index can again be calculated for each wavelength using equation (14). These values are listed as $\mathrm{n}_{2}$ in table 2 .

Table 2: Values of $\lambda, \mathrm{T}_{\max }$ and $\mathrm{T}_{\min }$ for the $\mathrm{PbTiO}_{3}$ transmittance spectra of figure 1; the underlined values of transmittance are the values calculated by suitable parabolic interpolation. The calculated values of refractive index and film thickness are based on the envelope method.

\begin{tabular}{cccccccccc}
\hline$\lambda(\mathrm{nm})$ & $\mathrm{T}_{\max }$ & $\mathrm{T}_{\min }$ & $\mathrm{T}_{\alpha}$ & $\mathrm{n}_{1}$ & $\mathrm{~d}_{1}$ & $\mathrm{~m}_{0}$ & $\mathrm{~m}$ & $\mathrm{~d}_{2}$ & $\mathrm{n}_{2}$ \\
\hline 434.5 & 0.531 & $\underline{0.483}$ & 0.507 & 3.185 & - & 7.702 & 7.5 & 511.523 & 3.119 \\
446.5 & $\underline{0.575}$ & 0.510 & 0.541 & 3.057 & - & 7.192 & 7 & 511.242 & 2.992 \\
471.5 & 0.654 & $\underline{0.546}$ & 0.597 & 2.898 & 422.325 & 6.458 & 6.5 & 528.702 & 2.934 \\
492.5 & $\underline{0.683}$ & 0.570 & 0.624 & 2.796 & 427.459 & 5.963 & 6 & 528.507 & 2.829 \\
524 & 0.730 & $\underline{0.585}$ & 0.654 & 2.734 & 538.304 & 5.482 & 5.5 & 526.993 & 2.759 \\
555 & $\underline{0.762}$ & 0.596 & 0.674 & 2.690 & 602.602 & 5.092 & 5 & 515.822 & 2.656 \\
601 & 0.807 & $\underline{0.638}$ & 0.718 & 2.529 & 494.994 & 4.421 & 4.5 & 534.674 & 2.589 \\
644.5 & $\underline{0.841}$ & 0.673 & 0.752 & 2.403 & 447.015 & 3.917 & 4 & 536.466 & 2.468 \\
711 & 0.895 & $\underline{0.696}$ & 0.790 & 2.323 & 531.010 & 3.432 & 3.5 & 535.730 & 2.382 \\
785 & $\underline{0.896}$ & 0.716 & 0.800 & 2.255 & 584.871 & 3.019 & 3 & 522.064 & 2.254 \\
883.5 & 0.890 & $\underline{0.721}$ & 0.801 & 2.235 & 679.027 & 2.658 & 2.5 & 494.028 & 2.114 \\
\hline
\end{tabular}

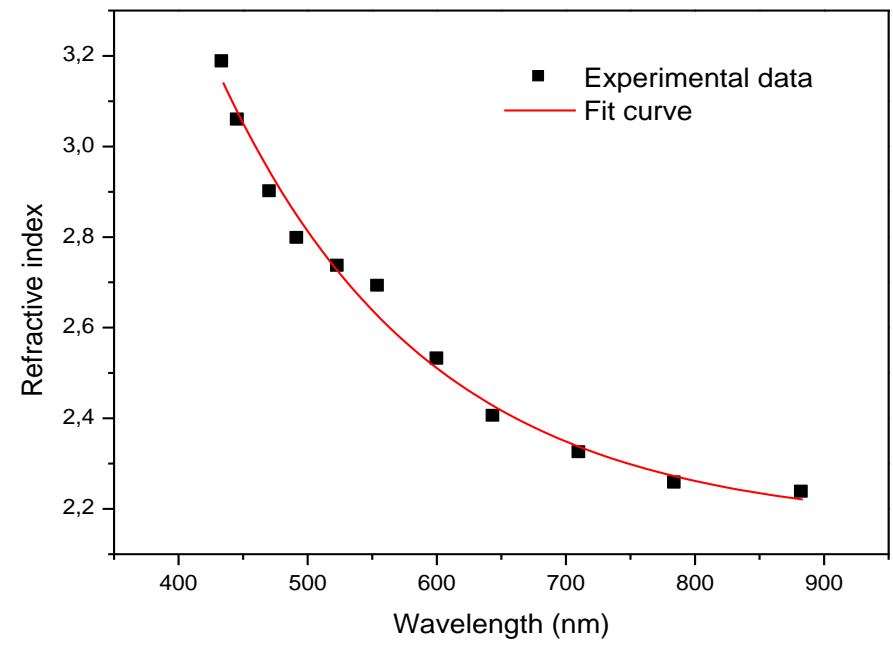

Figure 2: Refractive index dispersion spectra for $\mathrm{PbTiO}_{3}$ films deposited on $\mathrm{MgO}$ substrate

Figure 2 illustrates the dependence of the refractive index versus wavelength. In fact, the spectral behavior of refractive index for semi-conductor and dielectrics can be fitted by the one-oscillator Sellmeier dispersion equation [25]:

$$
n(\lambda)=n_{\infty}+\frac{a}{\lambda^{2}-b}
$$

Where a and $\mathrm{b}$ are constants and $n_{\infty}$ is the extrapolated refractive index in infrared. For our studied $\mathrm{PbTiO}_{3}$ thin film deposited on $\mathrm{MgO}$, using fit of the $\mathrm{n}_{2}$ values, we find $\mathrm{a}=14.66, \mathrm{~b}=160.60$ and $n_{\infty}=2.16$.

\subsection{Determination of optical absorption coefficient}


To determine the absorption coefficient, we used the transmittance $\mathrm{T}$ in region of higher absorption (between 350 and $600 \mathrm{~nm}$ ). The expression of $\mathrm{T}$ in this region is reduced to:

$$
T=\frac{A}{B} \exp (-\alpha d)
$$

Where $A=16 n^{2} s$ and $B=(n+1)^{3}\left(n+s^{2}\right)$

Equation (16) is valid provided that the extinction coefficient $\mathrm{k}$ is very low compared to refractive index $\mathrm{n}$, which is justified for our thin films in the considered spectral region. In this region of strong absorption, the interference fringes disappear and the three curves $T_{\max }, T_{\min }$ and $T_{\alpha}$ converge to a single curve. $A$ and $B$ depend on $n$ (refractive index of the thin film) and s (refractive index of the substrate). Since the thickness d is known and values of $n$ can be estimated by extrapolating the values calculated in the other part of the spectrum, thus we can determine the absorption coefficient $\alpha$ by the expression:

$$
\alpha=\frac{1}{d} \ln \left(\frac{A}{B T}\right)
$$

The results obtained are given in Figure 3. This latter figure shows the variation of the absorption coefficient as a function of wavelength. It seen that the absorption coefficient decreases when the wavelength increases.

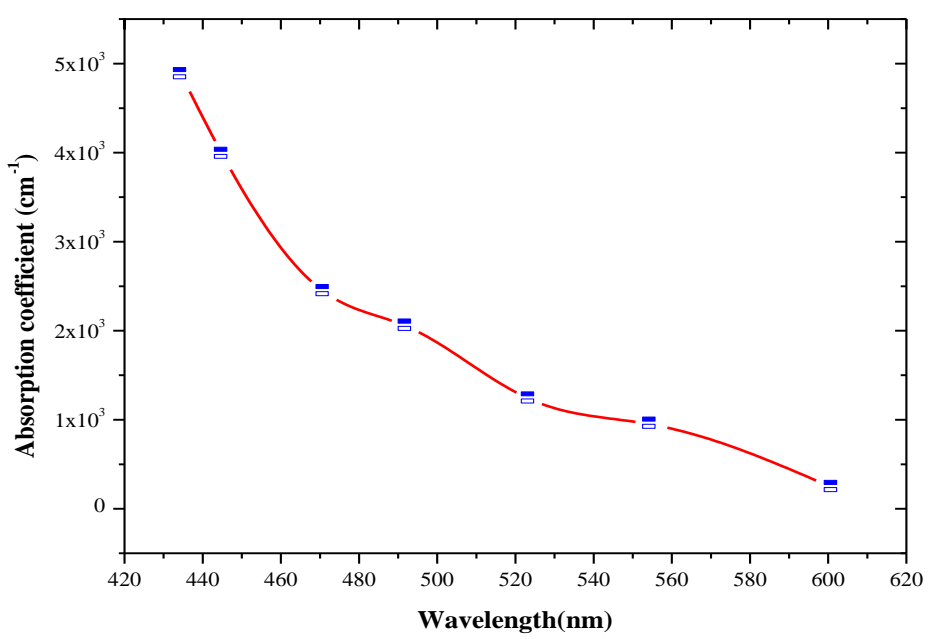

Figure 3: Absorption coefficient versus wavelength

Next, in order to complete the calculation of the optical constants, the extinction coefficient $k$ is estimated from the values of absorption coefficient $\alpha$ and wavelength $\lambda$ using the already mentioned formula:

$$
k=\frac{\alpha \lambda}{4 \pi}
$$

Figure 4 illustrates the dependence of extinction coefficient $\mathrm{k}$ versus wavelength for the studied thin film. This coefficient characterizes absorption of the electromagnetic wave energy in the process of propagation of a wave through a material. It also taken into account the scattering and luminescence effects.

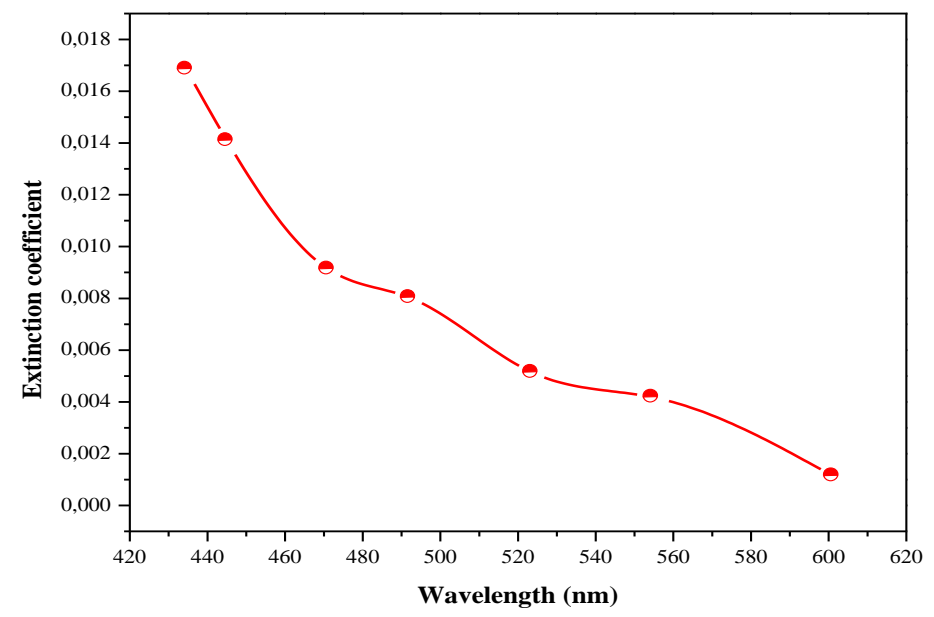




\subsection{Optical band gap}

Figure 4: Extinction coefficient versus wavelength

There are two types of optical transitions, which can occur at the fundamental absorption edge of crystalline as well as non-crystalline materials. They are direct and indirect transitions. When a light beam falls on a thin film, a part of the beam will be reflected, another part will be transmitted through the film, and the rest of the beam will be absorbed. Absorption of photons causes transition of the electrons from valance band to conduction band. The absorption ability is measured by its absorption coefficient $(\alpha)$ which is a function of frequency [2628]. The nature of the transition is determined by using the relation [25]:

$$
(\alpha h v)^{j}=\beta(h v-E g)(20)
$$

Where $h v$ is the photon energy, Eg presents the band gap energy, $\beta$ is a constant and it is function of refractive index. The constant $\mathrm{j}$ is equal to two (2) for electrons direct transition from valance band to conduction band and equal to one (1) for indirect transition [29].

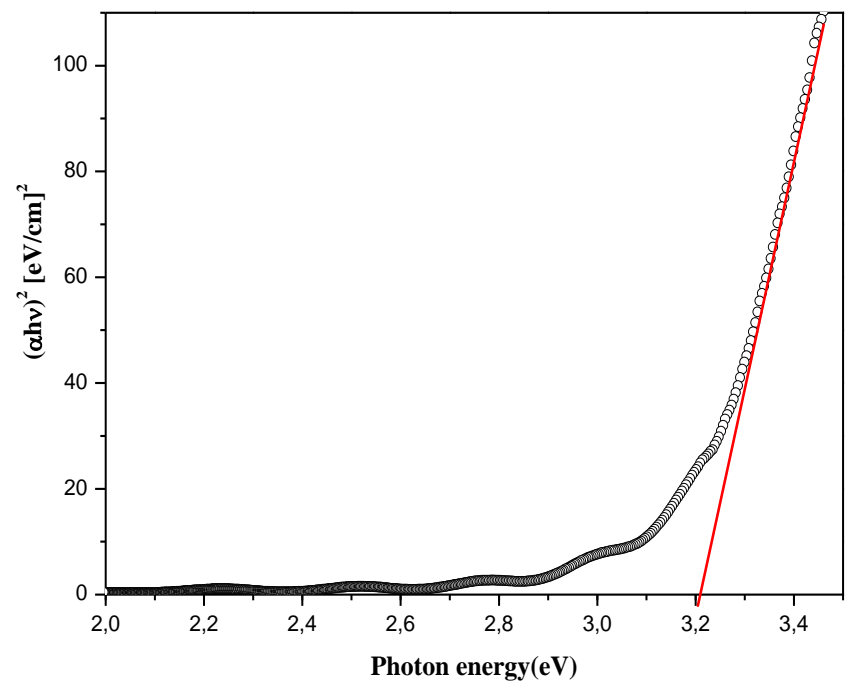

Figure 5: The dependence of $(\alpha h v)^{2}$ on photon energy in $\mathrm{PbTiO}_{3}$ deposited on $\mathrm{MgO}$ substrate

The band gap of the films has been calculated from the absorption edge of the spectrum. The PbTiO3 thin film considered as direct band gap materials [30]. Thus, band gap energy Eg can be estimated by assuming direct transition between conduction band and valance band. Thus the direct band gap can be evaluated by putting $\mathrm{j}=2$ in equation (20). The direct band gap is determined using this equation when linear portion of $(\alpha h v)^{2}$ against $h v$ plot is extrapolated to intersect the energy axis at $\alpha=0$ (Fig. 5)[31,32]. We have found that the value of the energy band gap of the PbTiO3 thin film deposited on $\mathrm{MgO}$ is $3.2 \mathrm{eV}$. This found value is in agreement with the review data reported previously [30]. The presence of a single slope in the plot suggests that the films have direct and allowed transition.

\section{Conclusion}

Lead titanate thin films have been deposited on $\mathrm{MgO}$ substrate by pulsed laser deposition. The optical properties of $\mathrm{PbTiO}_{3}$ thin films were analysed from the generation of the envelopes interference maxima and minima of the transmission spectrum. The dispersion refractive index $n(\lambda)$ found follows the one-oscillator Sellmeier law. However, the refractive indices are very slightly lower than those of the $\mathrm{PbTiO}_{3}$ bulk perovskite. We have found that both absorption coefficient $\alpha$ and extinction one k decrease as increasing the wavelength $\lambda$. The energy band gap is reasonable agreement with review data.

This thin films opto-geometrical characterization carried out by the method detailed in this article will allow a good knowledge of geometric (refractive index and thickness) and optical parameters (gap energy and absorption coefficient) for a use of the studied PT thin films in optical waveguide devices, dynamic random access memory (DRAM) materials, and electro-optical and sensor/actuator devices.

Acknowledgments-The authors wish to thank E. Millon, and A. Roemer from university of Metz (LSMCL laboratory) for their help concerning the thin films growth. 


\section{References}

1. Rui Guo, Lu You, Yang Zhou, Zhi Shiuh Lim, Xi Zou, Lang Chen, R. Ramesh \& Junling Wang, Nat. Comm. 4 (2013) 2990-2995.

2. J. C. Lashley, C. P. Opeil and J. L. Smith, Ferroelectric infrared detector and method, United States Patent No.: US 7,687,775 B2, Date of Patent: Mar. 30, 2010.

3. D. Akai, R. Yoshita and M. Ishida, J. Phys.: Conference Series 433 (2013) 012017(6pp).

4. T. Singh, A. Kumar and U. C. Naithani, Ind. J. Pure Ap. Phy. 48 (2010) 47-51.

5. T. C. King, J. J. Chen, K. C. Chang, and C. J. Wu, Appl. Optics 55 Issue 29 (2016) 8276-8279.

6. R. Nelmes and W. F. Kuchs, Solid State. Commun. 54(8) (1985) 721-723.

7. H. Li, J. Zhu, Q. Wu, J. Zhuang, H. Guo and Y. Ye, Ceram. Int. 43 (16) (2017) 13063-13068.

8. J. -Q. Dai, Y.-M. Song and H. Zhang, Physica B. 456 (2015) 383-387).

9. A. Roemer, A. Essahlaoui, O. Pons-Y-Moll, B. Vincent, R.M. Defourneau, W. Seiler, A. Boudrioua, E. Million, Thin Solid Films 453-454 (2004) 417-421.

10. B. Alshehri, S.-M. Lee, J.-H. Kang, S.-H. Gong, S.-W. Ryu,Y.-H. Cho, and E. Dogheche, Appl. Phys. Letter. 105(5) (2014) 51906 (4pp).

11. J. Rivory, Thin Solid Films 313-314 (1998) 333-340.

12. S. Funke1, B. Miller, E. Parzinger, P Thiesen1, A.W. Holleitner and U Wurstbauer, J. Phys.: Cond. Matter 28(38) (2016) 385301 (12pp).

13. K. A. Aly, H. H. Amer, A. Dahshan, Mater. Chem. Phys. 113 (2009) 690-695.

14. Y. Laaziz, A. Bennouna, M. Y. Elazhari, J. Ramiro-Bargueno, A. Outzourhit, N. chahboun and E. L. Ameziane, Thin Solid Films 303 (1997) 255-263.

15. B. Santic and F. Scholz, Measur. Sci. Tech. 19 (2008) 1-7.

16. A. Essahlaoui, H. Essaoudi, A. Hallaoui, A. Labzour, A. Housni, J. Mater. Environ. Sci.8 Issue 9 (2017) 3202-3207.

17. H. Tabata, T. Kawai, S. Kawai, O. Murata, J. Fujioka and S. Minakata, Appl. Phys. Lett. 59(19) (1991) 2354-2356.

18 C. M. Foster, Z. Li, M. Buckett, P. M. Baldo, L. E. Rehn, G. R. Bai, D. Guo, H. You, and K. L. Merkle, J. Appl. Phys. 78(4) (1985) 2607.

19 A. Essahlaoui, A. Roemer, A. Boudrioua, E. Million, J. C. Loulergue, Opt. Mater. 24(3) (2003) 465-470.

20 A. Roemer, A. Essahlaoui, O. Pons-Y-Moll, B. Vincent, R.M. Defourneau, W. Seiler, A. Boudrioua, E. Million, Thin Solid Films 453-454 (2004) 417-421.

21. Mi. A. Khan, T. P. Comyn and A. J. Bell, J. Eur. Ceram. Soc. 28(3) (2008) 591-597.

22. David M. Roessler and Donald R. Huffman, Handbook of Optical Constants of Solid

23. R. Swanepoel, J. Phys. E: Sci. Instrum. 17 (1984) 896-903.

24. J. C. Manifacier, J. Gasiot and J. P. Fillard, J. Phy. E: Sci. Instrum. 9 (1976) 1002-1004.

25. T. Bellunato, M. Calvi, C. Matteuzzi, M. Musy, D. L. Perego, B. Storaci, Europ. Phys. J. 52 (2007) 759764.

26. Y. E. Lee, Y. J. Kim and H. J. Kim, J. Mater. Res. 13(5) (1998) 1260-1265.

27. H. Tabet-Derraz, N. Benramdane, D. Nacer, A. Bouzidi and M. Medles, Sol. Energy Mater. Solar Cells. 73 (2002) 248-259.

28. L. F. Dong, Z. Cui and Z. K. Zhang, Nanostru. Mater. 8 (1997) 815-823.

29. N. Izyumskaya, V. Avrutin, X. Gu, U. Ozgur, T. D. Kang, H. Lee, D. J. Smith, H. Morkoc, Mater. Resear. Soci. 966 (2007) 217-222.

30. S. Piskunov, E. Heifets, R.I. Eglitis, G. Borstel, Compu. Mater. Sci. 29 (2004) 165-178.

31. A. Djelloul, M. Adnane,Y. Larbah, T. Sahraoui, C. Zegadi, A. Maha, B. Raha, J. Nano-and Elec. Phys. 7(4) (2015) 4045-4049.

32. C. Nassiri, A. Hadri, FZ. Chafi, A. El Hat, N. Hassanain, M. Rouchdi B. Fares1, A. Mzerd, J. Mater. Environ. Sci. 8 Issue 2 (2017) 420-425.

(2018) ; http://www.jmaterenvironsci.com 